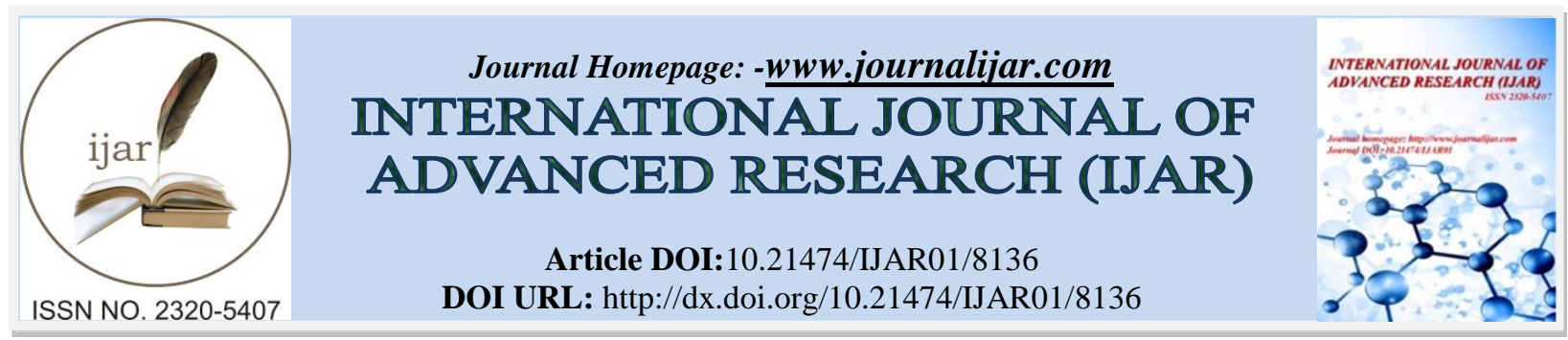

RESEARCH ARTICLE

\title{
A RARE POSTOPERATIVE NEUROLOGIC CRISIS.
}

\section{Afshan Punnepillil Thaha ${ }^{1}$, Sreekumar Ramachandran ${ }^{2}$, ShivanesanPitchai ${ }^{3}$ and Harishankar Ramachandran Nair ${ }^{4}$.}

1. MS, Senior Resident - Division of Vascular Surgery, Dept. of CVTS, SCTIMST.

2. MS, PDCC (Vascular Surgery), FRCS, Additional Professor- Division of Vascular Surgery, Dept. of CVTS, SCTIMST.

3. MS, MCh (Vascular Surgery), Assistant Professor - Division of Vascular Surgery, Dept. of CVTS, SCTIMST.

4. MS, MRCSEd, Senior Resident, MCh Vascular Surgery- Division of Vascular Surgery, Dept. of CVTS, SCTIMST.

\section{Manuscript Info}

(.........................

Manuscript History

Received: 01 October 2018

Final Accepted: 03 November 2018

Published: December 2018

Keywords:-

Akinetic crisis, Parkinson's disease, ruptured aortic aneurysm, aneurysm repair.

\section{Abstract}

Ischaemic myelopathy is one of the causes of limb weakness following open repair of aortic aneurysm. We present the case report of a 73 year old male, who was on treatment for Parkinsonism and became immobile after aortic surgery for ruptured infrarenal abdominal aortic aneurysm. Initially, ischaemic myelopathy was suspected, but it ultimately turned out to be acute presentation of akinetic crisis, which is a rare complication of Parkinson's disease. It is a preventable complication, as it most often occurs due to the inadvertent withdrawal of antiparkinsonian medications, as in our patient. Hence, it is important, especially for a vascular surgeon performing surgery for abdominal aortic aneurysm, to be aware of this condition for prompt diagnosis and treatment without which the condition causes serious morbidity and even mortality.

Copy Right, IJAR,2018, . All rights reserved.

\section{Introduction:-}

Parkinson's disease (PD) is a neurologic movement disorder which progresses slowly and ultimately leads to major disability ${ }^{1}$. Akinesia is one of the most common symptoms of Parkinson's disease ${ }^{2}$. Akinetic crisis seen in patients with Parkinson's disease is typified by severe disability to move with life threatening consequences and delayed responsiveness to treatment ${ }^{3}$.

Akinetic crisis is seen in high stress conditions like post- operative period following a major surgery like abdominal aortic aneurysm repair. The index patient was on treatment for Parkinson's disease and possibly omitted the drugs for the past few days. He presented with ruptured abdominal aortic aneurysm, underwent emergency open repair of the aneurysm, and developed akinetic crisis in the postoperative period.

\section{Case report}

73 year old male presented with sudden onset of abdominal pain, for four days, which worsened for past 8 hours. He was diagnosed to have abdominal aortic aneurysm three months back and was on followup elsewhere. He was also having gait disturbance for past six months, and was on treatment with levodopa and carbidopa, with a provisional 
diagnosis of Parkinsonism. The patient was admitted in outside hospital four days prior with worsening abdominal pain, decreased food intake and lethargy, and his medications were withheld since then. He was referred to us suspecting aneurysm rupture. Emergency computed tomographic aortogram was taken, which revealed, ruptured infrarenal aortic aneurysm to the left posterolateral aspect. He underwent emergency open repair of the aneurysm. From the first post operative day onwards, paucity of movement of limbs was noted, predominantly the lower limbs. MRI on the second postoperative day excluded ischaemic myelopathy. There was white matter atrophy involving the cerebral parenchyma. Patient's upper and lower limbs developed rigidity over the following days.

Considering the history of gait disturbances, and examination findings of rigidity involving all the limbs, we restarted levodopa-carbidopa on the second postoperative day, but with poor improvement of symptoms during the initial days. From the fifth postoperative day onwards, there was gradual resolution of his symptoms, he started to move upper limbs first, followed by lower limbs. The rigidity involving the limbs reduced and power increased to grade 4 . The patient was extubated on fourth post operative day and was slowly started on oral feeds, which he tolerated. He was gradually ambulated, although grade 1 rigidity persisted, predominantly in the lower limbs. He was discharged on 10th postoperative day with levodopa 100mg + Carbidopa $25 \mathrm{mg}$ thrice daily. He was doing well with only minimal motor symptoms at 3 months of follow up.

\section{Discussion:-}

The above case report is a relatively rare scenario of akinetic crisis which is a complication of Parkinson's disease where the motor symptoms have acutely worsened up to a point where the patient is almost completely immobile. It is often associated with hyperthermia, dysphagia, dysautonomia and increased levels of serum muscle enzymes ${ }^{4}$. It may resemble neuroleptic malignant syndrome in certain cases ${ }^{5}$. However, in the acute phase of this condition, dopaminergic drugs are found to be transiently ineffective ${ }^{4}$.

Dopaminergic and non-dopaminergic mechanisms of Parkinson's disease are involved in the clinical improvement of motor functions. But, there is a severe loss of presynaptic striatal dopamine function that does not improve even after recovery from akinetic crisis ${ }^{6}$. The exact mechanism that explains akinetic crisis is unknown. Triggering factors include treatment alterations or withdrawal, trauma, major surgery, infections or GI tract diseases ${ }^{4,7}$. Akinetic crisis does not seem to be related to disease severity of Parkinson's disease ${ }^{4}$, but rather a condition with unknown risk factors.

In the index patient, the triggering factor could have been withdrawal of drugs or an acute event in the abdomen, such as aneurysm rupture and ensuing surgery. One of the characteristic features of akinetic crisis appears to be the transient blockade of dopaminergic system that leads to long-lasting refractoriness to treatment by anti-parkinsonian medications ${ }^{(6)}$. In this case also, the patient did not respond to the anti-parkinsonian medications initially. Gradual improvement in the symptoms was noted from fifth post operative day onwards.

Akinetic crisis is often misdiagnosed and may present with or without fever ${ }^{6,8,9,10}$. Diagnostic clues include the clinical picture and the delay in response to treatment with rescue drugs or subcutaneous apomorphine infusion ${ }^{7}$. Even in our patient, the initial diagnostic suspicion was ischaemic myelopathy which can occur very rarely following abdominal aortic aneurysm repair, especially in the setting of rupture ${ }^{10}$.

This life-threatening complication of Parkinson's disease accounts for $15 \%$ deaths with an annual incidence of 0.3 $\%^{4,8}$. It is unlike the "wearing off phenomenon" that occurs when dopaminergic drug levels fall, and responds immediately to dopaminergic rescue drugs ${ }^{4}$.

The largest case series of patients with akinetic crisis in Parkinson's disease, published in 2003, studied 22 patients, and categorised the patients into 3 groups. Category 1: probable absorption deficit-akinetic crisis, Category 2: without deficit of absorption - pure akinetic crisis and Category 3: iatrogenic akinetic crisis-neuroleptic malignantlike syndrome. In all patients acute akinesia lasted for several days after tentative treatments, thus evidencing a transient unresponsiveness to dopaminomimetic agents ${ }^{7}$.

\section{Conclusion:-}

Although Parkinson's disease is a common neurological condition affecting elderly people causing significant morbidity, its implication in the postoperative setting has not been studied much. This case report highlights the 
situation where inadvertent withdrawal of anti-parkinsonian medications in a patient with Parkinson's disease, resulted in akinetic crisis. Physicians should be aware of this situation, as confusion in diagnosis may occur, especially in the postoperative setting, with other neurological conditions, as in this case where ischaemic myelopathy is a known complication of aortic aneurysm repair. Early recognition of the condition, and prevention, whenever possible should be the aim as it can lead to severe disability with life threatening consequences.

\section{References:-}

1. Magrinelli F, Picelli A, Tocco P, Federico A et al. Pathophysiology of Motor Dysfunction in Parkinson's Disease as the Rationale for Drug Treatment and Rehabilitation. 2016 Park Dis [Internet].

2. Onofrj M, Bonanni L, Cossu G, Manca D et al. Emergencies in Parkinsonism: akinetic crisis, life-threatening dyskinesias, and polyneuropathy during L-Dopa gel treatment. Parkinsonism RelatDisord. 2009 Dec 1;15:S233-6.

3. Onofrj M, Thomas A. Acute akinesia in Parkinson disease. Neurology. 2005 Apr 12;64(7):1162-9.

4. Toru M, Matsuda O, Makiguchi K, Sugano K. Neuroleptic malignant syndrome-like state following a withdrawal of antiparkinsonian drugs. J NervMent Dis. 1981 May;169(5):324-7.

5. Kaasinen V, Joutsa J, Noponen T, Päivärinta M. Akinetic Crisis in Parkinson's Disease Is Associated with a Severe Loss of Striatal Dopamine Transporter Function: A Report of Two Cases. Case Rep Neurol. 2014 Nov 26;6(3):275-80.

6. Thomas A, Iacono D, Luciano AL, Armellino K. Acute akinesia or akinetic crisis in Parkinson's disease. Neurol Sci. 2003 Oct 1;24(3):219-20.

7. Takubo H, Harada T, Hashimoto T, Inaba Y, et al. A collaborative study on the malignant syndrome in Parkinson's disease and related disorders. Parkinsonism RelatDisord. 2003 Apr;9 Suppl 1:S31-41.

Friedman JH, Feinberg SS, Feldman RG. A neuroleptic malignantlike syndrome due to levodopa therapy withdrawal. JAMA. 1985 Nov 15;254(19):2792-5.

8. Thomas A, Onofrj M. Akinetic crisis, acute akinesia, neuroleptic malignant-like syndrome, Parkinsonismhyperpyrexia syndrome, and malignant syndrome are the same entity and are often independent of treatment withdrawal. MovDisord Off J MovDisord Soc. 2005 Dec;20(12):1671-72.

9. Rosenthal D, Southern Association for Vascular Surgery. Spinal cord ischemia after abdominal aortic operation: Is it preventable? J Vasc Surg. 1999 Sep 1;30(3):391-9. 